the two years results were not consistant, the proportion of self-coloured roots being higher in 1962 , suggesting that the degree of pigmentation was liable to environmental modification. Fourth, in a few $F_{2}$ families and test crosses, the degree of pigmentation was so variable that discrete classes could no longer be recognised. Consideration of the genetic mechanisms of pigment distribution in the radish root is outside the scope of this report, but these results do establish, beyond doubt, that they are independent of the $H$ locus.

\title{
REFERENCES
}

HARBORNE, J. B. 1958a. Spectral methods of characterizing anthocyanins. Biochem. 7., $70,22-28$.

HARBORNE, J. B. 1958b. The chromatographic identification of anthocyanin pigments. 7. Chromat., $1,473-488$.

harborne, J. в. 1962a. The glycosidic pattern of anthocyanin pigments. Phytochemistry, 2,85 .

HARBORNE, J. B. 1962 $b$. Chemicogenetical studies of flavonoid pigments, chapter 18 in Chemistry of Flavonoid Compounds, ed. by T. A. Geissman. Pergamon Press, Oxford.

SCOTT-MONCRIEFF, R. 1936. A biochemical survey of some Mendelian factors for flower colour. 7. Genet., 32, 1 1 7-1 70 .

UPHоF, J. С. т. 1924. On Mendelian factors in radishes. Genetics, 9, 292-304.

\section{GENOTYPE FREQUENCIES IN A SECTION OF A CLINE}

\author{
P. HOLGATE
}

The Nature Conservancy, London, S.W.1

Received 7.ii.64

\section{INTRODUCTION}

The study of the geographical diffusion of genes through a population is considerably simplified if the population is thought of as being distributed continuously in a one-dimensional habitat, such as a stretch of coast or the banks of a stream. For such a population, consider a pair of alleles a and A at a given locus, and let the relative fitnesses of the genotypes aa, aA and AA be $\mathrm{I}+2 m, \mathrm{I}+m$ and $\mathrm{I}$, i.e. there is no dominance. Let $x$ be the distance from an arbitrary origin, and $p(x, t)$ the frequency of the $a$ gene in the population at position $x$ and time $t$. If the breeding system is one of discrete generations and local panmixia, after which the offspring wander at random in such a way that the distances they move are normally distributed with standard deviation $k$, then it is known that in the limit, as the generation time tends to zero, the diffusion of $a$ is represented by the differential equation

$$
\frac{\partial p(x, t)}{\partial t}=k \frac{\partial^{2} p(x, t)}{\partial x^{2}}+m p(x, t)\{\mathrm{I}-p(x, t)\} .
$$

Fisher (1937) discussed the case where the solution is a wave of stationary form advancing with constant velocity. A different approach is to suppose 
that the situation leading to (I) holds only in part of the habitat, say a finite or half infinite interval, and different selective factors operate elsewhere. As a consequence it is possible for a temporal equilibrium to be set up in which $p(x, t)$ is independent of $t$, but varies with $x$, and satisfies appropriate boundary conditions, giving rise to a cline. Haldane (1948) treated the case where an abrupt change in relative fitnesses occurred, and dominance was involved. Fisher (1950) dealt with the case involving a linear variation of selective advantage, and no dominance. Skellam (195I) treated the diffusion of individuals, rather than genes, through habitats in which conditions for their survival varied. In the present case, setting the term on the left of (I) equal to zero, dropping the $t$, and denoting derivatives with respect to $x$ by primes,

$$
k p^{\prime \prime}(x)=-m p(x)\{\mathbf{I}-p(x)\}
$$

\section{WAHLUND'S FORMULA}

Consider a population consisting of $\mathcal{N}$ equal, geographically separate sub-populations which do not intermate, in which the frequencies of the gene $a$ are $p_{i}(i=1, \ldots, n)$. The overall frequency $\bar{p}$, and the variance $v$ between sub-populations are given by

$$
\bar{p}=\mathcal{N}^{-1} \Sigma p_{i}, \quad v=\mathcal{N}^{-1} \Sigma p_{i}{ }^{2}-\bar{p}^{2}
$$

Wahlund (1928) showed that the overall proportions of $a a, a A$ and $A A$ genotypes were

$$
\bar{p}^{2}+v, \quad 2 \bar{p}(\mathrm{I}-\bar{p})-2 v, \quad(\mathrm{I}-\bar{p})^{2}+v .
$$

The effect, a reduction in the frequency of heterozygotes below what would occur in a homogeneous population, is the same as that arising from inbreeding with coefficient of inbreeding $F$, if $v$ is equated to $F p(1-p)$. Since the continuous diffusion model is obtained by a limiting process from a discrete time model in which mating and diffusion take place alternately, the limiting situation will be random mating between individuals which have the same value of $x$. Consequently, Wahlund's formula can be extended to cover this case, with the sums (3) replaced by integrals. If the population is uniformly distributed over $[0, b]$ these are:

$$
\bar{p}=b^{-1} \int_{0}^{b} p(x) d x, \quad v=b^{-1} \int_{0}^{b} p^{2}(x)-\tilde{p}^{2} .
$$

\section{GENOTYPE FREQUENCIES IN THE CLINE}

Suppose that (2) holds in the interval $[0, b]$. On integrating it over this interval, a relation between $v$ and $\bar{p}$ is obtained

$$
\begin{gathered}
k\left\{p^{\prime}(b)-p^{\prime}(\mathrm{o})\right\}=-b m\left(\bar{p}-v-\bar{p}^{2}\right) \\
v=\bar{p}(\mathrm{I}-\bar{p})-\frac{k}{b m}\left\{p^{\prime}(\mathrm{o})-p^{\prime}(b)\right\}
\end{gathered}
$$

where the appropriate one-sided derivatives are understood if $p^{\prime}(x)$ is discontinuous at o or $b$. This expresses the "variance" of the local frequencies in terms of the overall frequency, and on substituting in (4), which still holds 
in view of the limiting process, the overall frequencies of $a a, a A$ and $A A$ in the section of the habitat being considered are found to be

$$
\begin{gathered}
\bar{p}-\frac{k}{b m}\left\{p^{\prime}(\mathrm{o})-p^{\prime}(b)\right\}, \frac{2 k}{m}\left\{p^{\prime}(\mathrm{o})-p^{\prime}(b)\right\}, \\
\mathrm{I}-\bar{p}-\frac{k}{b m}\left\{p^{\prime}(\mathrm{o})-p^{\prime}(b)\right\} .
\end{gathered}
$$

\title{
4. SUMMARY
}

A situation is considered in which a cline is maintained by the random diffusion of a simply advantageous gene. It is pointed out that Wahlund's formula can be extended to cover this case, and it is applied to obtain overall genotype frequencies in terms of the overall gene frequencies, the selection and diffusion constants, and the rates of change of gene frequency at the boundaries of the section being considered.

\section{REFERENCES}

FISHER, R. A. 1937. The wave of advance of advantageous genes. Ann. Eugen., 7, 355-369.

FISHER, R. A. 1950. Gene frequencies in a cline determined by selection and diffusion. Biomstrics, 6, 353-36r.

haldane, J. B. s. 1948. The theory of a cline. 7. Genet., 48, 277-284.

SKELlAM, J. G. 1951. Random dispersal in theoretical populations. Biometrika, 38 , 196-218.

WAhLund, s. 1928. Zusammensetzung von Populationen und Korrelationserscheinungen von Standpunkt der Verebungslehre aus betrachtet. Hereditas, II, 65-106.

\section{NATURAL SELECTION THROUGH MATERNAL INFLUENCE}

\author{
ALFRED F. NAYLOR \\ Deportment of Genetics, McGill University, Montreal, Conada *
}

Received 8.iv. 64

\section{INTRODUCTION}

In discussions of natural selection at an autosomal locus (Haldane, r932; Hogben, I946; Li, I955) it is usual to assume that genotypes are selected because the zygotes have viabilitites or fecundities which depend on their own genotypes. One could suppose, however, that maternal genotypes at one locus might affect offspring fitnesses upon which offspring genotypes, however, have negligible effect. In mammals such an effect can be imagined to operate through intra-uterine environment or post-natal nutrition. Interestingly, Moriwaki and Tobari (1963) have demonstrated a genetic effect which operated through the mother on offspring fitness in Drosophila ananassa. It thus seems worthwhile to report some results in theory of selection through maternal influence.

* Present address: Perinatal Research Branch, National Institute of Neurological Diseases and Blindness, N.I.H., Bethesda, Maryland, U.S.A. 